\title{
Hyperbolic reaction-diffusion equations for a forest fire model
}

\author{
Vicenç Méndez and Josep E. Llebot \\ Grup de Física, Departament de Ciències Ambientals, Facultat de Ciències, Universitat de Girona, \\ Plaça Hospital 6, 17071 Girona, Catalonia, Spain
}

(Received 5 June 1997)

\begin{abstract}
Forest fire models have been widely studied from the context of self-organized criticality and from the ecological properties of the forest and combustion. On the other hand, reaction-diffusion equations have interesting applications in biology and physics. We propose here a model for fire propagation in a forest by using hyperbolic reaction-diffusion equations. The dynamical and thermodynamical aspects of the model are analyzed in detail. [S1063-651X(97)02912-7]
\end{abstract}

PACS number(s): 05.70.- a, 05.40. $+\mathrm{j}$

\section{INTRODUCTION}

An important problem of our society is the forest fire. Some important organizations, especially the USDA Forest Service, have been researching this theme for some time [1]. Their studies are based in very specific phenomena which take place in the forest fire [2].

On the other hand, recently several papers related with percolation theory and self-organized criticality (SOC) [3] are trying to provide a different dynamical model for the fire spread. The basic problem of the SOC models is their hard adaptation to the real problem. A common assumption of them is the incorporation of the reforestation concept in their restoration rules. This concept is a necessary condition in order for the system to reach self-organization. From the practical point of view this is not realistic because it is not admissible that burned trees may become green trees at the same time that green trees are burning.

The model we present in this work is not so specific as those employed by the ecologists but is more realistic than the SOC models, because our model may be applied in real time and the reforestation concept is not needed. Our aim is to propose a theoretical continuum-deterministic reactiondiffusion model in order to describe the dynamical evolution of the fire. Further generalizations of our model could be employed in realistic descriptions of the spread of fire but a connection between the characteristic parameters of our model and the experimental data is not yet established. At the moment we focus our attention on a simple model which will provide interesting information about dynamics and nonequilibrium thermodynamics of the forest fire propagation.

\section{THE MODEL}

In this section we build up the reaction-diffusion model from three reasonable hypotheses which are introduced gradually in order to analyze the effects of each one of them. We define $n$ as the normalized number of burning trees, $D$ as the diffusion coefficient, $\tau$ as the relaxation time or the delay time in the appearance of the fire flux, and $F$ as the reaction term between green and burning trees. If we want to investigate how $n$ changes with time and position, the evolution is given by the hyperbolic reaction-diffusion equation

$$
\tau \frac{\partial^{2} n}{\partial t^{2}}+\frac{\partial n}{\partial t}=D \frac{\partial^{2} n}{\partial x^{2}}+F(n)+\tau \frac{\partial F(n)}{\partial t}
$$

where $F(n)=r f(n)$ is the reaction term. This equation differs from the classical one in the term which includes the relaxation time $\tau$. This time describes the delay effect in the appearance of the fire when the first trees begin to burn. On the other hand, this equation has the interesting property that it describes traveling fronts which are constrained to propagate with a finite velocity. This and other properties are recently studied by Méndez and Camacho [4]. In fact, the study of propagation of fronts for parabolic reactiondiffusion equations has a long history going back to the works of Fisher [5] and Kolmogorov, Petrovsky, and Piskunov (KPP) in the 1930's [6].

In practice, Eq. (1) is used to describe the evolution of the system between two homogeneous steady states. In the forest fire model, both states are, as we shall see, the state corresponding to the whole forest green $(n=0)$ and the state corresponding to the whole forest burned $(n=1)$. Both states may be connected by a traveling front with a speed $v$ which must fulfill some restrictions. These came initially from the linearized theory (KPP method) but recently Benguria and Depassier [7] proposed new restrictions by means of variational formulation (BD method, for short) useful when linearization method does not hold. In order that Eq. (1) presents traveling fronts joining $n=0$ and $n=1$ it is necessary that [4]

$$
c_{L}^{(l)} \equiv \frac{2 \sqrt{f^{\prime}(0)}}{1+a f^{\prime}(0)} \leqslant c \leqslant \tilde{c} \equiv \frac{1}{\sqrt{a}}
$$

with $a \equiv r \tau<1$ and $c$ is the dimensionless velocity defined by $c=v / \sqrt{r D}$. These bounds follow from the linearization method (often called linear speed selection). However, when the linearization does not hold [for instance, when $f^{\prime}(0)$ $=0$ ], it is necessary to resort to another method, such as, for instance, the $\mathrm{BD}$ one (also called variational speed selection). From this method it follows that

$$
c^{(\mathrm{BD})} \equiv \frac{I}{\sqrt{a I^{2}+1}} \leqslant c \leqslant \widetilde{c} \equiv \frac{1}{\sqrt{a}},
$$


where

$$
I(g)=2 \frac{\int_{0}^{1} \sqrt{f g h} d N}{\int_{0}^{1} g\left(1-a f^{\prime}\right) d N},
$$

$h=-g^{\prime}>0$ with $g(N)$ an unknown auxiliary (trial) function. It is interesting to note that both speed selection methods predict the same maximum speed for the front $\widetilde{c}$ $=1 / \sqrt{a}$. This is, in fact, the speed of the characteristics corresponding to the hyperbolic nonlinear PDE given by the adimensional form of Eq. (1).

Our second assumption in this work is to construct an explicit form for the reaction term in Eq. (1). This term may be derived taking into account the interaction between the burning (denoted by $B$ ) and the green trees (denoted by $G$ ). This interaction is described by the irreversible reaction

$$
G+\beta B \stackrel{r}{\rightarrow}(\beta+1) B
$$

where $\beta(\geqslant 1)$ quantifies the number of burning trees needed in order to set fire to a near green tree. This parameter may be related, in practice, with the distance between trees and the capacity of a green tree to be burnt. So, for greater values of $\beta$ it is expected that the speed of the fire front will be smaller. The parameter $r$ is the reaction constant, which is inverse to the characteristic reaction time. So, $a$ is the quotient between the relaxation and reaction times. The reaction term is given as

$$
F\left(n_{G}\right)=r n_{G} n_{B}^{\beta}=r n_{B}^{\beta}\left(1-n_{B}\right),
$$

where $n_{G}+n_{B}=1$ (the total number of trees of the forest is constant and equal to the normalized value 1$), n_{G}$ and $n_{B}$ the number density of green and burning trees, respectively.

\section{CONSTRAINTS FOR THE SPEED OF THE FIRE FRONT}

With these two assumptions we find our two-dimensional (2D) reaction-diffusion equation. Introducing the new spatial and temporal variables $\mathbf{x}^{*}=\left(x^{*}, y^{*}\right)=\sqrt{r / D} \mathbf{x}, t^{*}=r t$ and $a=r \tau, n_{B} \equiv n$. Equation (1) together with Eq. (5) is written as

$$
a \frac{\partial^{2} n}{\partial t^{2}}+\frac{\partial n}{\partial t}=\nabla^{2} n+f(n)+a f^{\prime}(n) \frac{\partial n}{\partial t},
$$

where $f(n)=n^{\beta}(1-n)$ and $f^{\prime}(n)=d f / d n$. As we are interested in finding traveling fire fronts connecting two homogeneous steady states $n=0\left(n_{B}=0, n_{G}=1\right)$ and $n=1\left(n_{B}=1\right.$, $\left.n_{G}=0\right)$, we introduce the wave variable $z=\mathbf{k} \cdot \mathbf{x}-\omega t$, where $\mathbf{k}=\left(k_{1}, k_{2}\right)$ is the wave number vector and $\omega$ the frequency. The phase speed of the fire front is assumed to be the same that the speed of propagation of the interphase between the region where $n=1$ (all trees are burned) and $n=0$ (all the trees are green). This speed is found to be

$$
\mathbf{v}=\frac{\omega}{k} \frac{\mathbf{k}}{|\mathbf{k}|}
$$

and we may write the speed in terms of the dimensionless speed $c \equiv|\mathbf{c}|$ as $|\mathbf{v}|=|\mathbf{c}| \sqrt{r D}$. Defining $z^{*}=z \sqrt{r / D}$, we find $z^{*}=\mathbf{k}^{*} \cdot \mathbf{x}^{*}-\alpha t^{*}$, where

$$
\alpha=\frac{\omega}{r} \sqrt{\frac{r}{D}}=c_{x} \frac{k_{1}^{2}+k_{2}^{2}}{k_{1}}=c_{y} \frac{k_{1}^{2}+k_{2}^{2}}{k_{2}}=|\mathbf{c}||\mathbf{k}| .
$$

In terms of the $z^{*}$ variable we rewrite Eq. (6) as

$$
b N_{z z}+\alpha N_{z}\left[1-a f^{\prime}(N)\right]+f(N)=0,
$$

where $N\left(z^{*}\right)=n(\mathbf{k} \cdot \mathbf{x}-\alpha t)$ and $b=|\mathbf{k}|^{2}-a \alpha^{2}$ and we have omitted all the asterisks for notational simplicity. For $\beta=1$ (logistic reaction) the linearization theory holds [4]. We find that in this case it is possible to obtain fire fronts connecting $n=0$ (unstable state) to $n=1$ (stable state) evolving in time if its velocity of propagation is restricted to

$$
c_{L}^{(l)}=\frac{2 \sqrt{f^{\prime}(0)}}{1+a f^{\prime}(0)} \leqslant|\mathbf{c}| \leqslant \tilde{c}=\frac{1}{\sqrt{a}}
$$

with $a<1$ and $f^{\prime}(0)=1$. For $\beta=1$ linearization theory is no longer valid and we must apply another method. We use the BD method as an alternative one. Applying this method we obtain

$$
c_{L}^{(\mathrm{BD})}=\frac{I}{\sqrt{a I^{2}+1}} \leqslant|\mathbf{c}| \leqslant \tilde{c}=\frac{1}{\sqrt{a}},
$$

where $I(g)$ is given by Eq. (4). Let us now to apply the last method to a source term $f(N)=N^{\beta}(1-N)$. As the auxiliary function $g(N)$ must satisfy $g^{\prime}(N)<0$ we have, in principle, a wide range of $g$ functions to choose. We apply the method for two specific forms of $g$, also used by Benguria and Depassier. Taking

$$
g_{1}(N)=\left(\frac{1-N}{N}\right)^{\nu}
$$

one can perform the integrals

$$
\int_{0}^{1} \sqrt{f g h} d N=\sqrt{\alpha} \frac{\Gamma[1 / 2+(\beta-2 \nu) / 2] \Gamma(\nu+1)}{\Gamma(3 / 2+\beta / 2)}
$$

with $\nu<(\beta+1) / 2$,

$$
\int_{0}^{1} g(N) d N=\Gamma(\nu+1) \Gamma(1-\nu)
$$

with $\nu<1$, and finally

$$
\int_{0}^{1} g(N) f^{\prime}(N) d N=\nu \frac{\Gamma(\nu+1) \Gamma(\beta-\nu)}{\Gamma(\beta+1)} .
$$

So, from Eq. (4) one obtains

$$
\begin{aligned}
I= & 2 \sqrt{\nu} \frac{\Gamma[(\beta-2 \nu+1) / 2] \Gamma(\beta+1)}{\Gamma[(\beta+3) / 2]} \\
& \times \frac{1}{\Gamma(\beta+1) \Gamma(1-\nu)-a \nu \Gamma(\beta-\nu)}
\end{aligned}
$$


with $\nu<1$ and $\beta>1$. Taking now

$$
g_{2}(N)=\int_{N}^{1} f(x) d x
$$

one finds

$$
\begin{gathered}
\int_{0}^{1} \sqrt{f g h} d N=\frac{2}{3}\left[\int_{0}^{1} f(x) d x\right]^{3 / 2}, \\
\int_{0}^{1} g(N) d N=\int_{0}^{1} N f(N) d N, \\
\int_{0}^{1} g(N) f^{\prime}(N) d N=\int_{0}^{1} f(N)^{2} d N .
\end{gathered}
$$

So, from Eq. (4) we find

$$
I=\frac{4}{3} \frac{\left[\int_{0}^{1} f(N) d N\right]^{3 / 2}}{\int_{0}^{1}\left[N f(N)-a f(N)^{2}\right] d N} .
$$

Using the explicit form for the source term, we find after some algebra,

$$
\begin{aligned}
I= & \frac{4}{3} \frac{1}{\sqrt{(\beta+1)(\beta+2)}} \\
& \times \frac{(2 \beta+3)(\beta+3)(2 \beta+1)}{(2 \beta+1)(\beta+1)(2 \beta+3)-a(\beta+2)(\beta+3)} .
\end{aligned}
$$

As $I$, and as a consequence $c_{L}^{(\mathrm{BD})}$, depend on $\beta$ we may conclude that the minimum velocity necessary to make the existence of traveling wave fronts possible is constrained to the needed number of burning trees. In order to appreciate this effect we evaluate $c_{L}$ for $\beta=1$ and $\beta=2$ by using Eqs. (9) and (12) obtaining

$$
c_{L}^{(\mathrm{BD})}(\beta=1)=\frac{20}{9} \frac{\sqrt{6}}{5-2 a}
$$

and

$$
c_{L}^{(\mathrm{BD})}(\beta=2)=\frac{70}{9} \frac{\sqrt{3}}{21-4 a} .
$$

For any $a$ one concludes $c_{L}(\beta=1)>c_{L}(\beta=2)$ and the possible speed of the front may be lower for $\beta>1$ than for $\beta$ $=1$, as is expected because a great number of needed burning trees might decrease the speed of the fire propagation. Another interesting feature of the BD method is the maximum value for $c_{L}^{(\mathrm{BD})}$. As $I(g)$ takes different values for each trial function chosen then $I \in[0, \infty)$ and it is easy to show

$$
\tilde{c}=\max _{I \in[0, \infty)}\left(\frac{I}{\sqrt{a I^{2}+1}}\right) .
$$

\section{ADDITIONAL RESTRICTIONS FOR THE FIRE FRONT}

In this section we derive restrictions coming from the shape of the fire front. These are considered as restrictions on $N_{0}$ in terms of $a$ and $\beta$. If the fire spreads as a monotonic plane wave front there must exist an inflection point. Let $N_{0} \equiv N(x=y=t=0)=N(z=0)$ be the initial number of burning trees which generates the spread of the fire. If we force $z=0$ to be the inflection point, by rescaling the origin of $z$, we obtain from Eq. (7)

$$
\alpha N_{z}(z=0)\left[1-a f^{\prime}\left(N_{0}\right)\right]+f\left(N_{0}\right)=0 .
$$

Defining $p=-N_{z}$, it is easy to see that $p>0$ for $-\infty<z<$ $+\infty$ if the front is assumed, as in the literature, monotonically increasing with $z$, and $p$ reaches its maximum value just for $z=0$. So, from Eq. (13)

$$
p \leqslant p_{\max }=\frac{f\left(N_{0}\right)}{\alpha\left[1-a f^{\prime}\left(N_{0}\right)\right]}
$$

with

$$
f^{\prime}\left(N_{0}\right)<1 / a \text {. }
$$

On the other hand, introducing $p$ in Eq. (7) and integrating we find

$$
\alpha \int_{0}^{1} p\left[1-a f^{\prime}(N)\right] d N=\int_{0}^{1} f(N) d N .
$$

From Eqs. (14) and (16) we write the first additional restriction as

$$
\int_{0}^{1} f(N) d N \leqslant \frac{f\left(N_{0}\right)}{1-a f^{\prime}\left(N_{0}\right)} .
$$

In terms of the source term, Eq. (17) becomes the first restriction

$$
\frac{N_{0}^{\beta}\left(1-N_{0}\right)}{1-a\left[\beta N_{0}^{\beta-1}-(\beta+1) N_{0}^{\beta}\right]} \geqslant \frac{1}{(\beta+1)(\beta+2)}
$$

with $\beta>1$ and $0<N_{0}<1$ and from Eq. (15) the second restriction is

$$
\beta N_{0}^{\beta-1}-(\beta+1) N_{0}^{\beta}<\frac{1}{a} .
$$

These restrictions must be viewed as conditions on $N_{0}$ in order to have a monotonically increasing fire front in terms of the characteristics parameters $a$ and $\beta$ of the forest. Let us now specialize Eqs. (18) and (19) for $\beta=1$. The second restriction (19) is fulfilled if $a<1$. The first restriction (18) leads us to

$$
\frac{3-a-\sqrt{a^{2}+3}}{6} \leqslant N_{0} \leqslant \frac{3-a+\sqrt{a^{2}+3}}{6} .
$$


A sufficient condition in order for the first restriction to be fulfilled for any $a$ is $1 / 2-\sqrt{3} / 6 \leqslant N_{0} \leqslant 2 / 3$.

On the other hand, the steepness of the front is $p_{\max }$, and, therefore, the width of the front $L$ is the inverse of the steepness. So, we may define

$$
L=\frac{1}{|\mathbf{k}| p_{\max }}=c \frac{1-a f^{\prime}\left(N_{0}\right)}{f\left(N_{0}\right)} .
$$

For the classical case $(a=0), L=c / f\left(N_{0}\right)$ and taking $N_{0}$ $=1 / 2$ and the logistic source $(\beta=1)$ one recovers $L=4 c$ for the $1 \mathrm{D}$ model. In our case

$$
L=\frac{c}{N_{0}^{\beta}\left(1-N_{0}\right)}\left\{1-a\left[\beta N_{0}^{\beta-1}-(\beta+1) N_{0}^{\beta}\right]\right\} .
$$

It is expected that for an increasing value of $\beta$ (fixing $a, c$, and $N_{0}$ previously) the width be a monotonically increasing function, so the spatial region of mixed states between 0 and 1 must increase with the needed burning trees. To guarantee this behavior we impose that $d L / d \beta>0$. However, it is easy to prove, after some algebra, that a sufficient condition for $L$ to be a monotonically increasing function with $\beta$ is that $a$ $<1$ and $0<N_{0}<1$.

\section{EXACT SOLUTIONS}

We derive in this section exact solutions for the nonlinear differential equation (7). First of all we try to find solutions for the logistic reaction. Following [8], we assume an initial value $N(z=0)=1 / 2$ and a logistic reaction term of the form

$$
f(N)=\left\{\begin{array}{cc}
N, & 0 \leqslant N \leqslant \frac{1}{2} \\
1-N, & \frac{1}{2} \leqslant N \leqslant 1
\end{array}\right.
$$

with the conditions $N(z \rightarrow-\infty)=1$ and $N(z \rightarrow+\infty)=0$. For $0 \leqslant N \leqslant \frac{1}{2}, f(N)=N$ and Eq. (7) is written as

$$
b \frac{d^{2} N}{d z^{2}}+\alpha \frac{d N}{d z}(1-a)+N=0
$$

which is linear and has a solution of the form

$$
N(z)=A_{1} e^{m_{+} z}+A_{2} e^{m_{-} z}
$$

In order to have a monotonically decreasing front with $z$ it is necessary that the characteristic values $m_{ \pm}$are real, and this is fulfilled for

$$
|\mathbf{c}|=\frac{\alpha}{|\mathbf{k}|}>\frac{2}{1+a}
$$

which is exactly the same restriction coming from the linearization theory for the logistic case. On the other hand, both values of $m_{ \pm}$are negative, that is $N(z \rightarrow+\infty)=0$ is fulfilled if and only if $a<1$ and $c<1 / \sqrt{a}$. For $\frac{1}{2} \leqslant N \leqslant 1, f(N)=1$ $-N$ and Eq. (7) is

$$
b \frac{d^{2} N}{d z^{2}}+\alpha \frac{d N}{d z}(1+a)+1-N=0
$$

which has the solution

$$
N(z)=1+B_{1} e^{n_{+} z}+B_{2} e^{n_{-} z} .
$$

As $N(z \rightarrow-\infty)=1$ is fulfilled only for $n_{ \pm}>0$, it is necessary that $B_{2}=0$. On the other hand, it is necessary that $N(z=0)$ $=1 / 2$ and this implies that

$$
\begin{gathered}
A_{1}+A_{2}=\frac{1}{2}, \\
B_{1}=-\frac{1}{2} .
\end{gathered}
$$

Only one constant remains to be determined. This is done imposing that both curves must have the same slope, that is, $(d N / d z)_{z=0}$ must be equal for both solutions. This restriction becomes

$$
A_{1} m_{+}+A_{2} m_{-}=\frac{1}{2} n_{+}
$$

Solving the set of equations for the integration constants we get

$$
\begin{gathered}
A_{1}=\frac{\sqrt{c^{2}(1+a)^{2}-4}+2 c-\sqrt{c^{2}(1-a)^{2}+4}}{4 \sqrt{c^{2}(1+a)^{2}-4}}, \\
A_{2}=\frac{\sqrt{c^{2}(1+a)^{2}-4}-2 c+\sqrt{c^{2}(1-a)^{2}+4}}{4 \sqrt{c^{2}(1+a)^{2}-4}}
\end{gathered}
$$

and the solution is written as

$$
N(z)=\left\{\begin{array}{cl}
A_{1} e^{\frac{-c(1-a)+\sqrt{c^{2}(1+a)^{2}-4}}{2\left(1-a c^{2}\right)}} \frac{z}{k}+A_{2} e^{\frac{-c(1-a)-\sqrt{c^{2}(1+a)^{2}-4}}{2\left(1-a c^{2}\right)}} \frac{z}{k}, & z>0, \\
1-\frac{1}{2} e^{\frac{-c(1+a)+\sqrt{c^{2}(1-a)^{2}+4}}{2\left(1-a c^{2}\right)}} \frac{z}{k}, & z<0 .
\end{array}\right.
$$




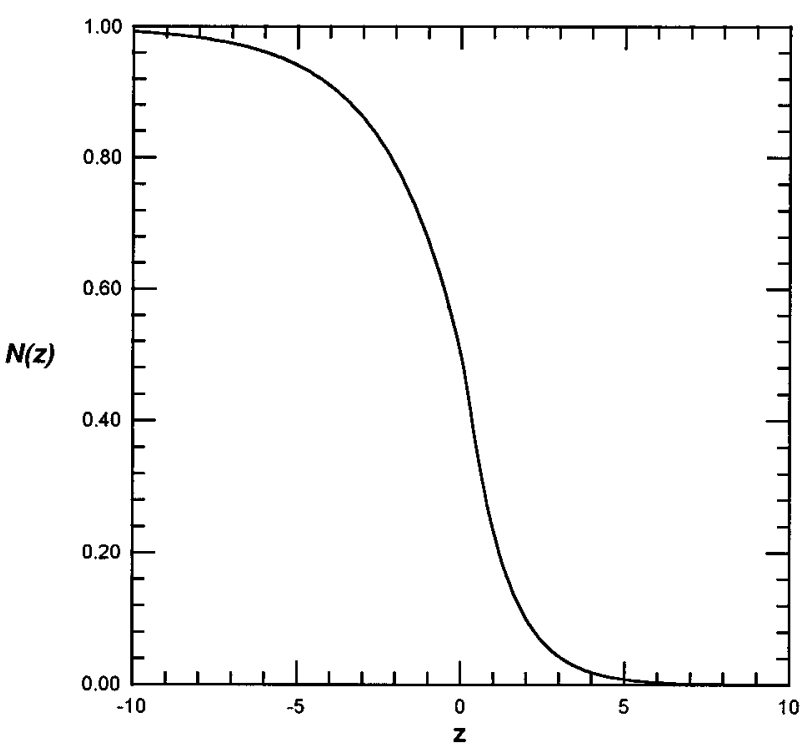

FIG. 1. Exact solution corresponding to Eq. (21) for $k=1, c$ $=1.8$, and $a=1 / 4$.

In Fig. 1 we plot for $k=1, c=1.8$, and $a=1 / 4$ the solution (21).

Now, we may also find exact solutions when the fire front travels with the maximum speed $|\mathbf{c}|=1 / \sqrt{a}$. In this situation, Eq. (7)

$$
\alpha N_{z}\left[1-a f^{\prime}(N)\right]+f(N)=0
$$

submitted to the restriction $f^{\prime}(N)<1 / a$, may be integrated to yield

$$
e^{\left(z-z_{0}\right) / \alpha}=f(N)^{a} \exp \left(-\int \frac{d N}{f(N)}\right)
$$

where $z_{0}$ is an integration constant to be determined from the initial conditions. For our source term, Eq. (22) reduces to

$$
\frac{z-z_{0}}{\alpha}=\ln \left[N^{a \beta-1}(1-N)^{a+1}\right]+\sum_{j=1}^{\beta-1} \frac{1}{(\beta-j) N^{\beta-j}} .
$$

For the logistic source $(\beta=1)$ this solution leads us to

$$
z=z_{0}+\alpha \ln \left[N^{a-1}(1-N)^{a+1}\right],
$$

where $z_{0}=2 a \alpha \ln 2$ for $N(z=0)=1 / 2$. For $\beta=2$ the corresponding solution is

$$
z=z_{0}+\alpha \ln \left[N^{2 a-1}(1-N)^{a+1}\right]+\frac{\alpha}{N} .
$$

Both cases may be depicted in Fig. 2. We can appreciate that the width of the front for $\beta=2$ is greater than for $\beta=1$. Only for $\beta=1$ and $a=1 / 3$ one can find an exact and invertible solution such as

$$
N(z)=1+\frac{1}{4} e^{3 z / 2 \alpha}-\frac{1}{4} \sqrt{e^{3 z / \alpha}+8 e^{3 z / 2 \alpha}}
$$

with $N_{0}=1 / 2$.

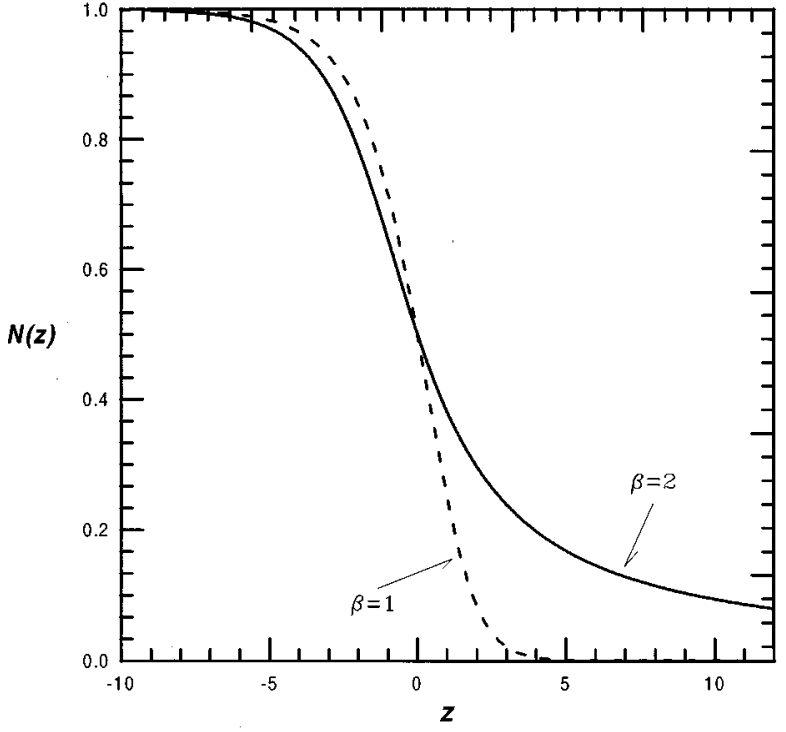

FIG. 2. Comparative plot between exact solutions for $\beta=1$ and $\beta=2$ for $a=1 / 3, c=\sqrt{3}, k=1 / \sqrt{3}$, and $N_{0}=1 / 2$.

\section{NONEQUILIBRIUM THERMODYNAMICS OF THE FIRE FRONT}

The steady states $n=0,1$ are thermodynamical equilibrium states. So, the fire front joins both equilibrium states, and this connection may be considered as a thermodynamical transport or a nonequilibrium process. In this sense we study in this section the thermodynamical description of the fire propagation. The extended irreversible thermodynamics [9] provides a thermodynamical interpretation when the entropy density for the burning trees depends on the classical variables as well as the dissipative fluxes. The consequent Gibbs equation is integrated to yield

$$
s^{(b)}=s_{\mathrm{eq}}^{(b)}-\frac{1}{2 T} \frac{\phi}{n^{(b)}} \mathbf{J} \cdot \mathbf{J},
$$

where $s_{\mathrm{eq}}^{(b)}$ is the local-equilibrium entropy density, $T$ the temperature, $\mathbf{J}$ the burning tree flux, $\mu$ a kind of chemical potential, and $\phi$ a parameter that will be identified later. Moreover we have from the Gibbs equation

$$
\left(\frac{\partial s^{(b)}}{\partial n^{(b)}}\right)_{\mathbf{J}}=-\frac{\mu}{T}
$$

and

$$
\left(\frac{\partial s^{(b)}}{\partial \mathbf{J}}\right)_{n^{(b)}}=-\frac{\phi \mathbf{J}}{n^{(b)} T} .
$$

From the particle balance equation for $n^{(b)}$

$$
\frac{\partial n^{(b)}}{\partial t}+\nabla \cdot \mathbf{J}=\mathbf{F}\left(n^{(b)}\right)
$$

and the balance equation for $s^{(b)}$ 


$$
\frac{\partial s^{(b)}}{\partial t}+\nabla \cdot \mathbf{J}^{(s)}=\sigma^{(b)}
$$

where $\mathbf{J}^{(s)}=-\mu \mathbf{J} / T$ is the entropy flux and $\sigma^{(b)}$ the entropy production as usual. It follows that

$$
\sigma^{(b)}=-\frac{\mathbf{J}}{T} \cdot\left(\nabla \mu+\frac{\phi}{n^{(b)}} \frac{\partial \mathbf{J}}{\partial t}\right)-\frac{\mu F}{T} .
$$

The physical volume element contains two subsystems. On one hand the burning trees-which are the foci of our attention - and, on the other hand, the media where these trees spread-which are the green trees. What must be positive definite is the total entropy production, that is, the entropy production of the whole forest $\sigma^{(f)}=\sigma^{(b)}+\sigma^{(g)}$. Thus we have

$$
\sigma^{(f)}=-\frac{\mathbf{J}}{T} \cdot\left(\nabla \mu+\frac{\phi}{n^{(b)}} \frac{\partial \mathbf{J}}{\partial t}\right)-\frac{\mu F}{T}+\sigma^{(g)} \geqslant 0 .
$$

The entropy production of the forest may be split into two contributions: that concerning the diffusion process $\left(\sigma^{(\mathrm{diff})}\right)$ and that corresponding to the reaction process $\left(\sigma^{(\mathrm{re})}\right)$

$$
\begin{gathered}
\sigma^{(\mathrm{diff})}=-\frac{\mathbf{J}}{T} \cdot\left(\nabla \mu+\frac{\phi}{n^{(b)}} \frac{\partial \mathbf{J}}{\partial t}\right) \geqslant 0, \\
\sigma^{(\mathrm{re})}=-\frac{\mu F}{T}+\sigma^{(g)} \geqslant 0 .
\end{gathered}
$$

The first equation is fulfilled by requiring the linear relation

$$
-\left(\nabla \mu+\frac{\phi}{n^{(b)}} \frac{\partial \mathbf{J}}{\partial t}\right) \equiv A \mathbf{J}
$$

with $A$ a positive scalar quantity. We define $\tau \equiv \phi / A n^{(b)}$ and $D \equiv\left(\partial \mu / \partial n^{(b)}\right) / A$. So from Eq. (26) one finds the transport equation

$$
\tau \frac{\partial \mathbf{J}}{\partial t}+\mathbf{J}=-D \nabla n^{(b)}
$$

From Eq. (27) and the balance equation for the burning trees number density (24) one obtains Eq. (1). Thus, we have shown that extended irreversible thermodynamics (EIT) provides hyperbolic reaction-diffusion equations for the forest fire as well as the thermodynamics quantities of the connecting process.

Let us now analyze in detail the nonequilibrium thermodynamics of the fire propagation. As is shown in [4], stochastic and EIT descriptions coincide for small fluxes. In order to calculate the chemical potential $\mu$ we use the stochastic description and we expand up to second order in $\mathbf{J}$. From the stochastic description for the entropy density [4] we find

$$
\frac{\mu}{k_{B} T}=-\frac{1}{k_{B}}\left(\frac{\partial s^{(b)}}{\partial n^{(b)}}\right)=n^{(b)}\left(1+\ln \frac{n^{(b)}}{2}\right)-\frac{\mathbf{J} \cdot \mathbf{J}}{2 n^{(b)}} .
$$

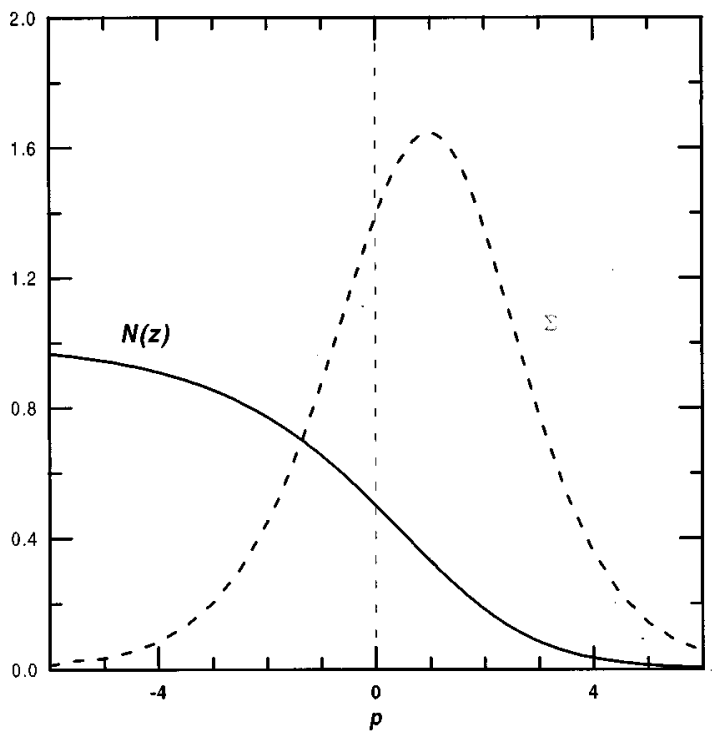

FIG. 3. Comparative plot between the exact solution (23) and the corresponding solution for the entropy production of the forest.

Introducing the dimensionless entropy production and flux $\sigma^{*}=\sigma / k k_{B}$ and $\mathbf{J}^{*}=\mathbf{J} \sqrt{\tau / D}$, respectively, we may write the entropy production of the burning trees as (omitting all the asterisks)

$$
\sigma^{(b)}=\frac{J^{2}}{a n^{(b)}}-f\left(n^{(b)}\right)\left[n^{(b)}\left(1+\ln \frac{n^{(b)}}{2}\right)-\frac{J^{2}}{2 n^{(b)}}\right] .
$$

If we assume that the entropy production of the reaction is zero because the burning and green trees have the same entropy production but with different sign, the entropy of the forest is positive definite and equal to

$$
\sigma^{(f)}=\frac{J^{2}}{a n^{(b)}} .
$$

Let us now compute the explicit solution for this entropy production for a specific situation. First of all we must calculate the flux J. Starting from Eq. (27) and defining $Y(z)$ $\equiv \mathbf{k} \cdot \mathbf{J}$ we may rewrite Eq. (27) in dimensionless form as

$$
\frac{d Y}{d z}-\frac{1}{a \alpha} Y=\sqrt{a \frac{d N}{d z}}
$$

and this may be integrated to yield

$$
Y=\sqrt{a} N+\frac{2}{3 \sqrt{a}} e^{2 p} \int N e^{-2 p} d p+C e^{2 p},
$$

where $p=3 z / 2 \alpha$ after integration by parts and $C$ is an integration constant such that $Y \rightarrow 0$ when $p \rightarrow \pm \infty$. The entropy production is given then by

$$
\Sigma \equiv|\mathbf{k}|^{2} \sigma^{(f)}=\frac{Y^{2}}{a N} .
$$

Using the explicit solution (23) we find 


$$
\begin{aligned}
Y(z)= & -\frac{\sqrt{3}}{12}\left(e^{3 z / 2 \alpha}+\frac{1}{6} e^{3 z / \alpha}\right) \\
& +\frac{\sqrt{3}}{36}\left(1+\frac{1}{2} e^{3 z / 2 \alpha}\right) \sqrt{e^{3 z / \alpha}+8 e^{3 z / 2 \alpha}}
\end{aligned}
$$

In Fig. 3 we plot $\Sigma$ in front of $p$ together with $N$. We observe that the entropy production reaches significant values in the step of the front, so, the thermodynamical region of interest is that of around $z=0$ because it is there where the nonequilibrium processes take place.

\section{CONCLUSIONS}

A hyperbolic reaction-diffusion equation for the forest fire propagation is studied as a model. To analyze the dynamical behavior of the fire front propagations, we use conventional tools, such as linearization and BD methods in order to find information about the speed of the front. Moreover, we have derived two additional restrictions coming from the shape of the fire front. These are established as conditions on the initial number of burning trees $N_{0}$ which generates the spread of the fire front, in terms of characteristics of the forest such as $a$ and $\beta$. We also study the width of the front $L$ which is always an increasing function of the necessary number of burning trees $\beta$ for the ignition of a neighboring green tree, if the front is stable $(a<1)$. Exact solutions are found but these are expressed as $N=N(z)$ only for the logistic reaction term.

Nonequilibrium thermodynamics for the connecting process between two equilibrium states were also studied. We calculated explicitly the entropy production of the forest and the burning trees flux. The entropy production of the forest is always positive and has two interesting properties: it has, in addition to the burning tree flux, a solitionlike form and reaches a maximum value just in the step of the front as expected.

\section{ACKNOWLEDGMENTS}

We acknowledge the support of the program Formación de Personal Investigador under Grant No. CLI95-1867 and the Spanish Ministry of Education and Science under Grant No. PB94-0718. The Statistical Physics Group of the Authonomous University of Barcelona is also acknowledged for the use of research facilities and we especially thank Professor D. Jou and Professor J. Casas-Vázquez.
[1] R. C. Rothermel (unpublished); F. A. Albini, G. N. Korovin, and E. H. Gorovaya (unpublished).

[2] A. M. G. Lopes, A. C. M. Sousa, and D. X. Viegas, Num. Heat Transfer, Part A 27, 229 (1995).

[3] B. Drossel and F. Schwabl, Phys. Rev. Lett. 69, 1629 (1992); M. Paczuski and P. Bak, Phys. Rev. E 48, R3214 (1993); K. Christensen, H. Flyvbjerg, and Z. Olami, Phys. Rev. Lett. 71, 2737 (1993); B. Drossel and F. Schwabl, Physica A 199, 183 (1993); Phys. Rev. E 50, 1009 (1994); Phys. Rev. Lett. 76, 936 (1996).
[4] V. Méndez and J. Camacho, Phys. Rev. E 55, 6476 (1997).

[5] R. A. Fisher, Ann. Eugenics 7, 355 (1937).

[6] A. Kolmogorov, I. Petrovsky, and N. Piskunov, Bull. Univ. Moscow, Ser. Int. A 1, 1 (1937).

[7] R. D. Benguria and M. C. Depassier, Phys. Rev. Lett. 77, 1171 (1996).

[8] D. S. Jones and B. D. Sleeman, Differential Equations and Mathematical Biology (Allen \& Unwin, London, 1983).

[9] D. Jou, J. Casas-Vázquez, and G. Lebon, Extended Irreversible Thermodynamics, 2nd ed. (Springer, Berlin, 1996). 